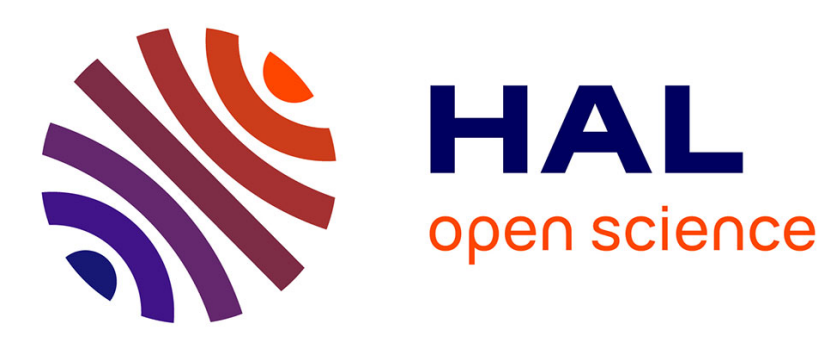

\title{
DAFS Study of Strained III-V Epitaxial Semiconductors
}

M. Proietti, Hubert Renevier, J. Berar, V. Dalakas, J. Hodeau, G. Armelles, J. García

\section{To cite this version:}

M. Proietti, Hubert Renevier, J. Berar, V. Dalakas, J. Hodeau, et al.. DAFS Study of Strained IIIV Epitaxial Semiconductors. Journal de Physique IV Proceedings, 1997, 7 (C2), pp.C2-749-C2-751. 10.1051/jp4:1997225 . jpa-00255304

\section{HAL Id: jpa-00255304 https://hal.science/jpa-00255304}

Submitted on 1 Jan 1997

HAL is a multi-disciplinary open access archive for the deposit and dissemination of scientific research documents, whether they are published or not. The documents may come from teaching and research institutions in France or abroad, or from public or private research centers.
L'archive ouverte pluridisciplinaire HAL, est destinée au dépôt et à la diffusion de documents scientifiques de niveau recherche, publiés ou non, émanant des établissements d'enseignement et de recherche français ou étrangers, des laboratoires publics ou privés. 


\title{
DAFS Study of Strained III-V Epitaxial Semiconductors
}

\author{
M.G. Proietti, H. Renevier*, J.F. Berar*, V. Dalakas*, J.L. Hodeau*, G. Armelles** and J. García \\ Instituto de Ciencia de Materiales de Aragón, CSIC-Universidad de Zaragoza, pza. S. Francisco s.n., \\ 50009 Zaragoza, Spain \\ * CNRS-UJF, Laboratoire de Cristallographie, 38042 Grenoble cedex, France \\ ** Instituto de Microelectrónica de Madrid, CSIC, Serrano 144, 28006 Madrid, Spain
}

\begin{abstract}
The effect of built-in strain on III-V epitaxial semiconductors has been investigated by Diffraction Anomalous Fine Structure (DAFS). We study two different systems in a different strain regime: a Strained Layer Superlattice of $(\mathrm{GaP})_{2}(\operatorname{InP})_{3}$ grown on a $\mathrm{GaAs}(001)$ substrate, and a single epilayer of $\mathrm{GaAs}_{1-\mathrm{x}} \mathrm{P}_{\mathrm{x}},(\mathrm{x}=0.225)$, also grown on a $\mathrm{GaAs}(001)$ substrate. In the first case the strain is accommodated by plastic deformation of the lattice, while in the second one it is partially relaxed by dislocations generation. The bond distances for the $\mathrm{Ga}-\mathrm{P}$ and $\mathrm{Ga}-\mathrm{As}$ pairs are obtained showing how they are affected by strain. The Ga-As pair shows to be "softer" than the Ga-P pair, i.e. more available to accommodate strain by bond deformation, in good agreernent with previous results obtained by different techniques. The DAFS provide a unique tool of studing systems that are out of the reach of the other X-ray techniques.
\end{abstract}

\section{INTRODUCTION}

Mismatched heteroepitaxy of III-V semiconductors is a subject of great interest due to its important applications in the field of electronic and optoelectronic devices [1]. Despite of the interest and efforts spent on the growth and characterization of this class of materials, there are only a few works presenting direct measurements of their structural parameters.

EXAFS would be the most suitable technique to get information about the short-range structure of these compounds but it cannot be applied in a straightforward way due to the peculiar nature of the epitaxial samples: too much thin to be measured in transmission and often grown on a substrate having some of the atomic components in common with the epilayer, giving rise to a large background signal. Alternative approaches, as glancing-angle EXAFS or SEXAFS, have been used [2,3] but they solve the problem only in part, since the signal collection is restricted to a few monolayers below the surface, allowing a quasi-surface measurements of very thin layers. The aim of this work is to study the structural properties of different strained III-V semiconductors samples using the alternative approach given by the DAFS spectroscopy. This spectroscopy, based on the principle that absorption-like information can also be obtained from scattering measurements, can combine the advantages of X-Ray Diffraction (XRD) and XAFS [4]. It provides XAFS-like information about a long-range order subset of atoms selected by diffraction (site selectivity). Like XAFS, DAFS is chemically and valence specific (chemical selectivity). The advantage of DAFS for studying this kind of systems, is that it allows to get the structural information from the epilayer, as a whole, just by choosing the correspondent Bragg peak (site-selective Bragg peak) of the strained phase.

The samples we measured represent two different regimes of strain. In one case the sample is a Strained Layer Superlattice (SLS) of $(\mathrm{GaP})_{2}(\mathrm{InP})_{3}$ grown on a (001) GaAs substrate. The subindexes 2 and 3 refere to the number of atomic monolayers (ML) constituting the individual Gap and InP layers. The overall SLS thickness is $2500 \AA$. Both components present a large mismatch $(-3.6 \% \mathrm{GaP},+3.8 \% \mathrm{InP})$ with $\mathrm{GaAs}$ giving rise to a large biaxial deformation in the individual layers. Nevertheless the strain is of opposite sign, i.e. tensile for GaP and compressive for InP, so that its net value in the SIS as a whole, is very smail, favoring the structure stability. In the second case the sample is an epilayer of $\mathrm{GaAs}_{1-\mathrm{x}} \mathrm{P}_{\mathrm{X}}$, with $\mathrm{x}=0.225,5000 \AA$ thick, grown on a GaAs (001) substrate as well. Due to the high thickness the strain tends to relax by generating misfit dislocations and cracks. The strain anyhow is not yet completely relaxed since XRD measurements show the presence of a "residual" strain of about the $0.5 \%$. In this way we can compare, among other things, measuring the DAFS at the Ga k-edge, how the strain can affect the same kind of bond, Ga-P, in different systems.

\section{EXPERIMENTAL AND DATA ANALYSIS}

The samples have been grown on semi-insulating GaAs (001) oriented substrates by Atomic Layer Molecular Beam Epitaxy (ALMBE). The growth details are reported elsewhere [5]. The XRD spectra, taken on the (004) and (115) Bragg reflections, to measure the lattice parameters perpendicular and parallel to the surface, show the epilayer Bragg peak well visible and separated from the substrate contribution. The DAFS measurements have been performed at the beamline D2AM at ESRF (Grenoble, France), that is dedicated to anomalous scattering studies. The DAFS spectra were recorded at room-temperature, at the Ga Kedge $(10367 \mathrm{eV})$ for both samples, on the (006) Bragg reflection. A top-DAFS -scan procedure was used for recording the spectra. It consists in measuring the diffracted intensity at the top of the reflections while scanning the beam energy. In the present study the structural information could be obtained with single site-selective Bragg reflections, i.e. the reflections belonging to the epilayer. In such a case there is no requirement to know the crystallographic structure [6,7].The DAFS spectra were analysed following a Kramers-Kronig iterative procedure $[6,7]$ to obtain the real, $f$, and imaginary, $\mathrm{f}^{\prime}$, anomalous parts of the complex atomic scattering factor of $\mathrm{Ga}$. It has to be noted that the absorption correction is negligible for the low 
thicknesses of the samples studied, in any case the Kramers-Kronig iterative procedure allows to correct the spectra for absorption as well (that is actually the only way to do it in case it is needed). Once $\mathrm{f}^{\prime}$ and $\mathrm{f}^{\prime \prime}$ are obtained, the data are processed according to the standard EXAFS data analysis procedures.

The raw DAFS spectra are shown, together with the correspondent $f$ and $f^{\prime \prime}$ components, in Fig. 1
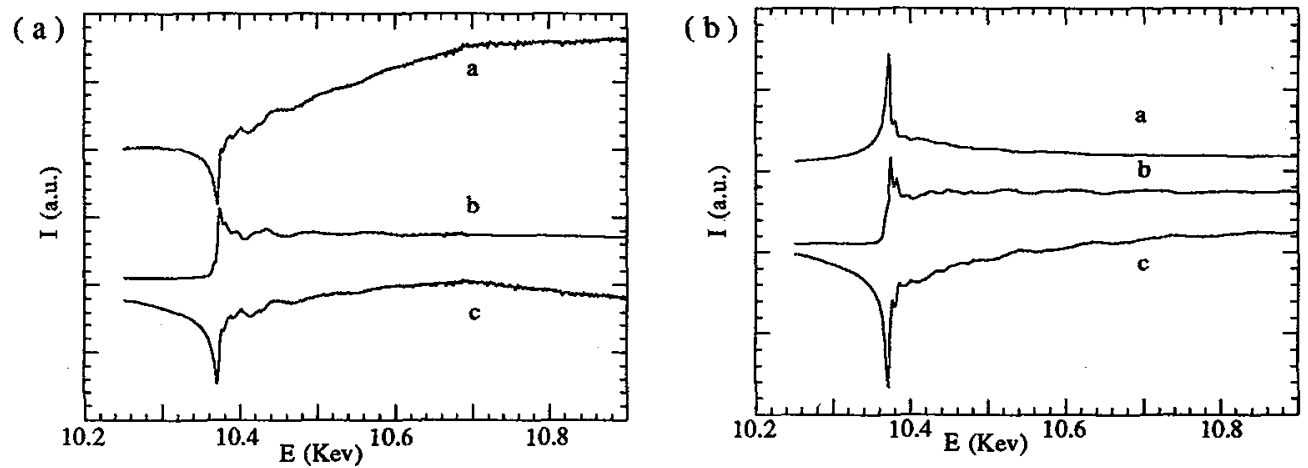

Fig.1:a) DAFS spectra at Ga k-edge, of the 006 Bragg reflection; b) Real part (f) of the Ga atomic scattering factor; c) Imaginary part $\left(f^{\prime \prime}\right)$ of the scattering factor. Panel (a) referes to the SLS sample and panel (b) to the GaAsP epilayer.

The EXAFS spectra have been extracted from the $\mathrm{f}^{\prime \prime}$ spectra shown in fig.1, according to the standard background removal techniques and are shown in fig.2a) together with the EXAFS spectra of the experimental models of GaAs and GaP bulk samples measured in transmission mode. The corresponding FT, are reported in fig. $2 \mathrm{~b}$ ). The $\mathrm{k}$ range used was from 2.5 to $11.9 \AA^{-1}$ for the SLS, and from 2.8 to $9.5 \AA^{-1}$ for the GaAsP epilayer.

(a)

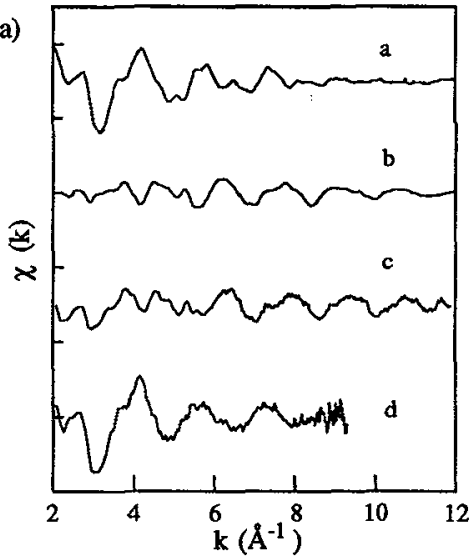

(b)

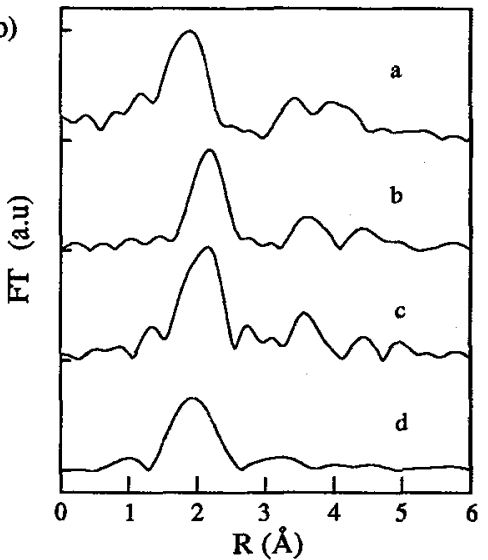

Fig.2: Panel a) EXAFS spectra and Panel b) FT spectra, of bulk GaP (a), bulk GaAs (b), GaAsP epilayer (c), and SLS (d).

\section{RESULTS AND CONCLUSIONS.}

The EXAFS spectra of the two samples (curves $c$ and d) are quite different from each other as expected from the different backscattering elements, mostly As for the GaAsP epilayer and only $P$ for the SLS. In the case of GaAsP the EXAFS spectrum looks clearly as a mixing of the GaAs and GaP model spectra, while for the SLS it's similar to the GaP model but definitely less structured. No appreciable second shell contribution is observed in the SLS FT spectrum. In order to get quantitative information from the EXAFS data, we have performed a least-square data analysis of the first and second shell of the GaAsP epilayer and of the first shell for the SLS. Data were Fourier filtered to isolate the contribution of the first and second (only for the GaAsP sample) shell. The results of the best-fit procedure are reported in Table 1 and the best-fit curves, for the first shell, are shown in fig.3, compared with the experimental filtered signais.

The results for the SLS show a change $\Delta R=0.04 \AA$ for the Ga-P bondlength. It seems to be slightly higher than the value previewed by the elastic theory [8], although the difference $(0.01 \AA)$ it is still within the uncertainty on the determination of $R$. On the other side deviations from the elastic approximation have been observed [9] for highly strained systems, as InAs/InP, with layer thicknesses lower than $10 \AA$ (3-4ML). As mentioned above, the II shell contribution for the SLS is quite small and a second shell analysis is not possible. We want to notice that this is not due to a poor signal-to-noise ratio, as can be 
observed in fig.2. The reason is instead the mixing, as Next Nearest Neighbors (NNN), of Ga and In atoms whose phases interferes destructively, producing a severe reduction (about a factor of 2 in theoretical simulations) of the NNN contribution.

Table 1: Best fit values obtained for coordination numbers $(N)$, interatomic distances $(R)$ and Debye-Waller factors (difference with the model ) $\Delta\left(\Delta \sigma^{2}\right)$, for the two samples studied. The errors on the fit parameters are $\Delta N / N=20 \%, \Delta R=0.01 \AA$ for the first shell," $\Delta \mathrm{R}=0.02 \AA$ for the II shell, $\Delta(\Delta) \sigma^{2}=0.002 \AA^{2}$ and $\Delta \mathrm{E}_{0}=0.1 \mathrm{eV}$ in both cases. We also report the known crystallographic $\mathrm{R}$ values for the GaAs and GaP bulk models, the values obtained by XRD (for the GaAsP sample), the values measured for diluted bulk alloys[10], and those deduced by the elastic theory [ 8 ].

\begin{tabular}{|c|c|c|c|c|c|c|c|}
\hline Sample & pair & exp./theor. & shell & $\mathbf{N}$ & $\mathbf{R}(\mathrm{A})$ & $\Delta(\Delta) \sigma^{2}(\AA)^{2}$ & $E_{0}$ \\
\hline$(\mathrm{GaP}) /(\operatorname{InP}) \mathrm{SLS}$ & Ga-P & DAFS & I & 4.0 & 2.40 & -0.003 & -0.1 \\
\hline (1) & $"$ & bulk alloy & I & - & 2.38 & - & - \\
\hline$"$ & $"$ & elastic & I & - & 2.39 & - & - \\
\hline GaAsP epilayer & Ga-As & DAFS & I & 3.6 & 2.40 & -0.002 & 1.3 \\
\hline${ }^{1}$ & Ga-P & DAFS & I & 1.2 & 2.36 & 0.002 & 1.6 \\
\hline$"$ & Ga-As & bulk alloy & I & - & 2.43 & - & \\
\hline$"$ & Ga-P & bulk alloy & I & - & 2.38 & - & - \\
\hline$"$ & $\mathrm{Ga}-\mathrm{Ga}_{\mathrm{a}}$ & DAFS & II & 18 & 3.94 & 0.002 & 1. \\
\hline$"$ & Ga-Ga & XRD & II & - & 3.96 & - & - \\
\hline$"$ & $\mathrm{Ga}-\mathrm{Ga}$ & elastic & II & 18 & 3.98 & - & - \\
\hline GaAs bulk & Ga-As & XRD & I & 4 & 2.448 & - & - \\
\hline$"$ & $\mathrm{Ga}-\mathrm{Ga}$ & XRD & II & 12 & 3.997 & $=$ & - \\
\hline GaP bulk & Ga-P & XRD & I & 4 & 2.361 & - & - \\
\hline 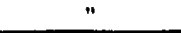 & $\mathrm{Ga}-\mathrm{Ga}$ & XRD & II & 12 & 3.856 & - & - \\
\hline
\end{tabular}

(a)

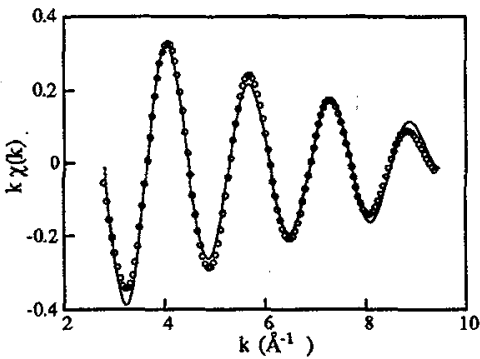

(b)

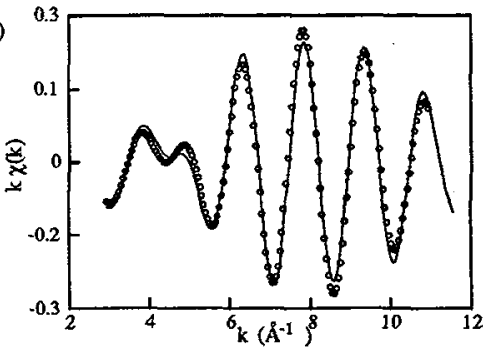

Fig.3: a) best-fit curve of the I shell contribution, (---), and filtered $k \chi(k),(\infty o o)$, for the SLS sample. b) best-fit curve of the I shell contribution, (---), and filtered $k \chi(k)$, (ooo), for the GaAsP sample.

The results for the GaAsP epilayer show a value of $2.36 \AA$ for the Ga-P bondlength, i.e. equal to the distance in bulk GaP, and a distance of $2.40 \AA$ for the Ga-As pair, that is shorter than the crystallographic value for GaAs, $2.448 \AA$. These values can be compared with the bondlength deformation obtained for diluted alloys of GaAsP in ref.10. In that case the maximum deformation observed is of $-0.022 \AA$ for the Ga-As and of $0.021 \AA$ for the Ga-P pair, for very low concentration, less than $10 \%$, of the pair considered. In this case it is instead the Ga-As bond which accommodates the deformation in terms of bond compression. It means that the residual built-in strain still acts on the lattice. The Ga-As bond is in this sense more able than Ga-P to adapt to strain, as expected from the lower elastic constants of GaAs. This is in very nice agreement with Raman measurements performed on the same sample [11], showing that the strain-induced shift of the Raman peaks is higher for the GaAs-like vibration than for the GaP-like mode. The only way to explain the result is to assume that the built-in strain affects more the Ga-As bond than the Ga-P bond. The result about the II shell of coordination shows that the NNN distance is lower than that previewed by the elastic approximation, as expected due to the release of strain occurred in the sample. The value we obtain coincides with the value deduced by diffraction, giving a further confirmation of the reliability of the DAFS results.

\section{References}

[1] See for example, T.R. Chen, Y.H. Zhuang, L.E. Eng, and A. Yariv, Appl. Phys Lett. 57, 2402 (1990); Jae-Hoon Kim, A. Larsson, and L.P. Lee, Appl. Phys. Lett.58, 7 (1991).

[2] M.G. Proietti, S. Turchini, J.Garcia, G. Lamble, F. Martelli, T. Prosperi, J. Appl. Phys 78, 6574 (1995).

[3] M.G. Proietti et al. on these same proccedings.

[4] H. Stragier, J.O. Cross, J.J. Rehr, L.B. Sorensen, Phys. Rev. Lett., 21,3064 (1992).

[5] F. Briones, L. Gonzalez, and A. Ruiz, Appl. Phys A 49, 729 (1989).

[6] I.J. Pickering, M. Sansone, J. Marsch, and G.N. George, J.Am. Chem Soc. 115, 6302 (1993).

[7] H. Stragier, Ph.D. Thesis, Universiry of Washington (1993).

[8] J. Hornstra and W.J. Bartels, J. of Cryst. Growth 14, 518 (1979).

[9] E. Bergignat, M. Gendry, G. Hollinger, and G. Grenet, Phys. Rev. B 49, 13544 (1994).

[10] J.B. Boyce and J.C. Mikkelsen, J. Cryst. Growth 98, 37 (1989).

[11] G. Armelles, M. L. Sanjuán, L.Gonzalez, and Y. Gonzalez, Appl. Phys. Lett. 68, 1805 (1996). 